\title{
Core Stabilization Exercise Improves Pain Intensity, Functional Disability and Trunk Muscle Activity of Patients with Clinical Lumbar Instability: a Pilot Randomized Controlled Study
}

\author{
Pattanasin Areeudomwong, PhD candidate, PT ${ }^{1)}$, Rungthip Puntumetakul, PhD, PT ${ }^{1,2)}$, \\ Kitti Jirarattanaphochai, PhD, MD ${ }^{3)}$, Sawitri Wanpen, $\mathrm{PhD}, \mathrm{PT}^{1,2)}$, \\ Jaturat Kanpittaya, MD ${ }^{4)}$, Uraiwan Chatchawan, PhD, PT ${ }^{1,2)}$, \\ Junichiro Yamauchi, $\mathrm{PhD}^{5,6)}$ \\ 1) School of Physical Therapy, Faculty of Associated Medical Sciences, Khon Kaen University: \\ 123, Mitraphab Highway, Muang District, Khon Kaen, 40002 Thailand. TEL: +66 83-419-6186, \\ FAX: +66 43 2020856, E-mail: rungthip@kku.ac.th \\ 2) Back, Neck and Other Joint Pain Research Group, Khon Kaen University \\ 3) Department of Orthopaedics, Faculty of Medicine, Khon Kaen University \\ 4) Department of Radiology, Faculty of Medicine, Khon Kaen University \\ 5) Graduate School of Human Health Sciences, Tokyo Metropolitan University \\ 6) Future Institute for Sport Sciences
}

\begin{abstract}
Purpose] To determine the therapeutic effects of core stabilization exercise in the treatment of patients with clinical lumbar instability. [Methods] Twenty subjects with clinical lumbar instability were randomly assigned to a treatment or control group. The treatment group received 10 weeks of core stabilization exercise (CSE), while the control group (CG) received 10 weeks of hydrocollator therapy and trunk stretching exercises. Pain intensity of the instability catch sign (ICS), functional disability, and trunk muscle activation patterns were measured before and at one day after 10 weeks of intervention. [Results] Both groups showed a significant reduction in pain intensity of ICS and reduced functional disability after 10 weeks of intervention; however, a significant improvement in the ratio activation of the transversus abdominis and internal oblique muscle relative to the rectus abdominis was only seen in CSE. In the comparison between groups, CSE showed significantly greater improvement in reduction of pain intensity during the ICS, significantly reduced functional disability, and significantly increased deep abdominal muscle activation after 10 weeks of intervention compared to CG. [Conclusion] The 10 weeks of CSE provided better therapeutic effects for pain intensity of ICS, reduced functional disability, and facilitated deep abdominal muscle activation of patients with clinical lumbar instability.

Key words: Clinical lumbar instability, Core stabilization exercise, Trunk muscle activation patterns
\end{abstract}

(This article was submitted Apr. 25, 2012, and was accepted Jun. 5, 2012)

\section{INTRODUCTION}

Low back pain (LBP) continues to be a major health problem and economic burden worldwide ${ }^{1)}$. In Thailand, the prevalence of LBP ranges from $46.6 \%$ to $68.6 \%{ }^{2,3}$, and the costs of management of LBP are relatively high, at approximately US 1,000 dollars per person, annually ${ }^{4)}$. The heterogeneous condition of LBP is categorized into subgroups. One subgroup of LBP is clinical lumbar instability and it is defined as the inability of the spine to maintain its normal pattern of displacement under physiologic loads. Clinical lumbar instability does not have initial or additional neurologic deficits, major deformity or incapacitating pain ${ }^{5)}$. Clinical lumbar instability is assessed by signs and specific clinical tests, such as painful catch $^{6}$, apprehension sign ${ }^{6}$, prone instability test ${ }^{7)}$ and the instability catch sign (ICS) ${ }^{6,8)}$.
Clinical lumbar instability can worsen without appropriate treatment, and patients may develop more structural degeneration with remarkable instability ${ }^{9)}$.

Core stabilization exercise (CSE) has become one of the therapeutic approaches for LBP, and it aims to enhance neuromuscular control skill, through preferential re-training of the deep abdominal and deep back muscles around the lumbar spine. It has been suggested that CSE can improve dynamic stability of the lumbar spine ${ }^{10)}$. Previous studies have revealed the effectiveness of CSE in patients with $\mathrm{LBP}^{11,12)}$. Kumar ${ }^{13)}$ also showed that CSE has an immediate effect on joint mobility and the pain pressure threshold of patients with clinical lumbar instability. However, there is currently no study available of the assessment of pain intensity of ICS, functional disability and trunk muscle activation patterns after a short period of exercise inter- 
vention.

The purpose of this study was to evaluate the therapeutic effects of core stabilization exercise performed for 10 weeks on pain intensity of ICS, functional disability and trunk muscle activation patterns in patients with clinical lumbar instability. We hypothesized that, because CSE can enhance the preferential activation of the deep abdominal and deep back muscles around the lumbar spine, it might improve pain of ICS and reduce the functional disability of patients with clinical lumbar instability.

\section{SUBJECTS AND METHODS}

A prospective, parallel group, assessor-blind, pilot study was conducted at the Associated Medical Sciences Laboratory, Khon Kaen University, Thailand. The study was approved by the Ethics Committee for Human Research of Khon Kaen University.

Patients with clinical lumbar instability were recruited from the Orthopaedic Outpatient Department, the Physical Medicine and Rehabilitation Outpatient Department and the Physical Therapy Department of Srinagarind Hospital, Khon Kaen Province, Thailand. They were screened by interviewing for history, physical examination, and using anteroposterior, lateral and flexion-extension radiographs to exclude other spinal conditions by an orthopaedic surgeon who was unaware of the treatment and assessment procedures. Eligible patients were invited to participate in this study and they gave their written informed consent before participation. Inclusion criteria were patients aged between 20-60 years and who had LBP for at least three months, with or without radiating leg pain. In addition, all patients included in this study presented the instability catch sign $(\mathrm{ICS})^{6,8)}$ with pain intensity measured on a numerical rating scale (NRS) of at least five out of 10, and also presented a positive sign in provocation testing, with at least one positive sign from painful catch $^{6}$, apprehension sign ${ }^{6}$ or the prone instability test ${ }^{7}$. The patients were ineligible if they had serious spinal pathology (e.g. spinal fracture, spinal malignancy or spinal infection), neurological signs (at least two of the following signs: weakness of the lower extremities, reflex changes or abnormality or loss of sensation of the lower extremities associated with the spinal nerve root) ${ }^{14)}$, obvious abnormal movement of the lumbar motion segment when assessing flexion-extension radiographs (sagittal plane translation larger than $4.5 \mathrm{~mm}$ of vertebral body width according to others, or sagittal plane rotation larger than 15 degrees at L1-L2, L2-L3 or L3-L4, 20 degrees at L4-L5 or 25 degrees at $\mathrm{L} 5-\mathrm{S} 1)^{5)}$ or were regularly receiving CSE, hydrocollator therapy or trunk stretching exercises.

Twenty subjects were recruited (10 female, 10 male) and randomly assigned to either the core stabilization exercise (CSE) or control group (CG) using a block randomized allocation with a block size of two. Subjects chose an opaque, sealed envelope which contained their group information. The randomization process was undertaken by the first researcher who was not involved in the recruitment of the subjects, treatments or evaluation processes. Subjects who received CSE or CG were supervised by the second researcher.

Subjects performed CSE which was modified from the treatment approaches of Koumantakis et al. ${ }^{15)}$ and Richardson and Jull ${ }^{10)}$. All subjects performed two 20-minute sessions of the exercise per week for 10 weeks. This exercise aims to enhance neuromuscular control skill and retrain deep abdominal and deep back muscles around the lumbar spine $^{10)}$. CSE was divided into three stages: 1) each subject was given instructions on how to correctly isolate low-load activation of the transversus abdominis (TrA) and lumbar multifidus (LM) muscles using an abdominal drawing-in maneuver (ADIM) technique in minimal loading positions such as prone lying and sitting. They were trained until they were able to perform these exercises with a 10 -second hold for 10 contraction repetitions, and were then trained in co-activation of TrA and LM (weeks 1 and 2). A pressure biofeedback device (Chattanooga Australia Pty. Ltd., Brisbane, Queensland) and electromyography (MP 100, BIOPAC Systems, Goleta, California) biofeedback were provided to the subjects to facilitate correct performance of each muscle contraction. 2) Once subjects could accurately perform contraction of TrA and LM, the exercises progressed to integration with controlling movements of the extremities and heavier loading positions with a 10 -second hold for 10 contraction repetitions (weeks 3-7). 3) Then, during weeks $8-10$, they performed functional tasks and pain-aggravating activities such as walking, lifting, running and cleaning the floor at work intensities that did not provoke pain.

The subjects had their exercise performance re-assessed every session to determine whether they could successfully perform the previous exercise. If the subjects failed to accurately perform the previous exercise, they were retrained in the previous exercise until they could perform.

The subjects in CG performed stretching exercises for the trunk muscles, and hydrocollator therapy. The stretching exercises were a single knee brought to the chest from the crook lying position and side bending in the standing position with contralateral arm elevation. These exercises did not exceed normal trunk range of motion and were performed at intensities that did not provoke pain. The exercises lasted 20 minutes, with two sessions per week for 10 weeks. Subjects performed five minutes of trunk stretching exercises once a day with a 10-second hold and 10 repetitions of each exercise. In addition, they were treated for 15 minutes with a hydrocollator $\left(60^{\circ} \mathrm{C}\right)$ which was placed beneath the painful area of their lower back in the supine lying position immediately after trunk stretching exercises.

Both groups were required to daily perform a set of home exercises of the same level, position and frequency as those demonstrated during the exercise session. During the intervention period, the second researcher made a phone call to motivate the subjects in both groups every week to continue their daily home exercises.

Pain intensity during the ICS (primary outcome), the Roland-Morris Disability Questionnaire Thai version, and trunk muscle activation patterns (secondary outcomes) were measured before and at one day after 10 weeks of intervention by the third researcher who was blinded to the experimental procedures. 
Pain intensity of ICS was measured using an 11-point NRS (0-10 scale) scale ${ }^{16}$. The subjects were asked to circle the number on the NRS scale that best described the pain intensity of ICS (pain during return from trunk flexion to standing), where zero indicated no pain anywhere and 10 indicated the worst pain ever experienced.

The Roland Morris Disability Questionnaire (RMDQ) Thai version is a condition-specific functional measure designed to assess physical disability due to LBP. There are 24-items. The subjects put a tick against the item that describe them on the evaluation day. If an item does not describe them, then they leave the space blank and go on to the next item. The scores range from zero (no disability) to 24 (maximum disability) ${ }^{17)}$.

Four-channel surface electromyography (sEMG) was used to continuously record EMGs. The sampling rate was $1,000 \mathrm{~Hz}$ with signal amplification of gain $\times 1,000$ and a common mode rejection ratio (CMRR) of $110 \mathrm{~dB}$. The signal frequency was band-pass filtered between 10 and $500 \mathrm{~Hz}$. A personal computer with an A/D converter was used to record and analyze the EMG data. The raw data generated during sub-maximal voluntary isometric contraction (sub-MVIC) was used instead of MVIC since sub-MVIC is better able to detect changes in levels of motor activity during the performance of low load tasks than $\mathrm{MVIC}^{18)}$.

The skin over the boundaries of the right side of transversus abdominis (TrA) and internal oblique (IO) (TrA \& IO), rectus abdominis (RA), lumbar multifidus (LM) and iliocostalis lumborum pars thoracis (ICLT) muscles were prepared to reduce skin impedance to less than $5 \mathrm{k} \Omega$ by shaving hair at the electrode sites, cleaning the sites with alcohol, and abrading the skin with fine sandpaper. After that, pairs of active disc disposable $\mathrm{Ag} / \mathrm{AgCl}$ surface electrodes (EL 503) with electrical contact surface areas of $1 \mathrm{~cm}^{2}$ and a center-to-center spacing of $2.5 \mathrm{~cm}$ were attached parallel to each muscle on the right side as previously reported: TrA \& IO muscle ${ }^{19)}$, RA muscle ${ }^{20)}, \mathrm{LM}$ muscle ${ }^{21)}$ and ICLT muscle ${ }^{22)}$. The two ground electrodes of the right RA and ICLT muscles were placed over the ribcage and the other two ground electrodes of the right TrA \& IO and LM muscles were placed over the right iliac crest. Snap leads were used to connect the surface electrodes with the amplifier to transfer signals. All data of sub-MVIC and ADIM were analyzed using the root mean square (RMS) technique.

To generate sub-MVIC for the abdominal muscles, each subject performed a double foot raise of $1 \mathrm{~cm}$ from the floor in the crook lying position three times with five-second holds ${ }^{18)}$, and the RMS values of TrA \& IO and RA muscles were simultaneously recorded. For sub-MVIC testing of back muscles, each subject performed a double knee raise of $5 \mathrm{~cm}$ from the floor in the prone lying position with $90^{\circ}$ of knee flexion, three times with five-second holds, and the RMS values of LM and ICLT muscles were simultaneously recorded. Two minutes rest was given between the tests to avoid muscle fatigue ${ }^{23)}$. The normalized RMS data of the middle three seconds of the five-second testing period were analyzed and the average values of the three tests were used as reference values of sub-MVIC normalization for each muscle $^{24)}$.

The ADIM was performed three times with 10 -second holds, and a one minute rest period between the tests was given to avoid muscle fatigue $\left.{ }^{23}, 25\right)$. To generate ADIM, each subject gently drew in the lower anterior abdominal wall below the navel level with no movement of the spine and pelvis, and a small pillow was placed under the ankles in the four-point kneeling position ${ }^{10)}$. The $\mathrm{sEMG}$ was used to record the right TrA \& IO, RA, LM and ICLT muscle activations. The middle five seconds of normalized RMS data from the 10 second testing period were analyzed and the average values of three tests were used as reference values of ADIM for each muscle ${ }^{25}$.

To compute the activation ratio of the abdominal muscles, the normalized data of the TrA \& IO and LM muscles were divided by the normalized data of the RA (TrA \& IO/RA ratio) and ICLT (LM/ICLT ratio) muscles ${ }^{26)}$.

Data are presented as means and standard deviations (SD). All continuous data were normally distributed, as tested by the Kolmogorov-Smirnov test. The paired t-test was used to test within-group differences between before and at one day after 10 weeks of intervention. Analysis of covariance (ANCOVA) was used to compare the magnitude of the effects of the intervention between the two groups, and to estimate the adjusted mean differences and the $95 \%$ confidence intervals $(95 \% \mathrm{CI})$ for each outcome measure of each group, using a baseline score as a covariate variable. All analyses were performed on the basis of intention-to-treat with the last observation carried forward for the subjects who did not complete the full study schedule.

\section{RESULTS}

The characteristics of the subjects of both groups are shown in Table 1. The baseline values of the pain intensity instability catch sign (ICS), functional disability and activation ratio of LM relative to ICLT muscles (LM/ ICLT ratio) were similar among groups $(\mathrm{p}=0.08, \mathrm{p}=0.22$ and $\mathrm{p}=0.79$, respectively); however, the ratio of $\operatorname{TrA} \&$ IO relative to RA muscles was not ( $\mathrm{TrA} \& \mathrm{IO} / \mathrm{RA}$ ratio) $(p=0.04)$. At the end of the 10 weeks of CSE, $60 \%$ of the subjects were in the third stage, $20 \%$ were in the second stage and $20 \%$ remained in the first stage.

Analysis of differences within each group at one day after 10 weeks of intervention revealed that both groups had significant differences in decreased pain intensity of ICS (CSE: mean difference $=4.60 \pm 2.12,95 \% \mathrm{CI}=3.08$ to $6.12, \mathrm{p}<0.001 ; \mathrm{CG}$ : mean difference $=1.10 \pm 1.10,95 \% \mathrm{CI}$ $=0.31$ to $1.88, \mathrm{p}=0.012$ ), and in functional disability scores (CSE: mean difference $=5.90 \pm 3.25,95 \% \mathrm{CI}=3.58$ to 8.22 , $\mathrm{p}<0.001 ; \mathrm{CG}$ : mean difference $=1.90 \pm 2.64,95 \% \mathrm{CI}=0.01$ to $3.79, \mathrm{p}=0.049$ ). Furthermore, within group comparison of trunk muscle activation patterns showed that the CSE group had a significantly higher $\operatorname{TrA} \&$ IO/RA ratio at one day after 10 weeks of intervention than the baseline value (mean difference $=-4.31 \pm 1.42,95 \% \mathrm{CI}=-5.32$ to $-3.30, \mathrm{p}<0.001$ ), while CG showed a significant decrease (mean difference $=$ $4.21 \pm 2.47,95 \% \mathrm{CI}=2.45$ to $5.98, \mathrm{p}<0.001)$. However, there was no significant in the LM/ICLT ratio in either group. 
1010 J. Phys. Ther. Sci. Vol. 24, No. 10, 2012

Table 1. Demographic characteristics of the subjects

\begin{tabular}{|c|c|c|}
\hline Characteristics & Core stabilization exercise $(\mathrm{n}=10)$ & Control group $(\mathrm{n}=10)$ \\
\hline Age (years), mean (SD) & $38.9(16.2)$ & $39.0(8.8)$ \\
\hline Gender, n (\%) of female & $5(50)$ & $5(50)$ \\
\hline Height (cm), mean (SD) & $158.9(8.4)$ & $156.6(8.8)$ \\
\hline Weight (kg), mean (SD) & $52.9(7.3)$ & $61.9(14.7)$ \\
\hline Body mass index $\left(\mathrm{kg} / \mathrm{m}^{2}\right)$, mean $(\mathrm{SD})$ & $20.9(2.6)$ & $23.18(4.9)$ \\
\hline \multicolumn{3}{|l|}{ Occupation, n (\%) } \\
\hline Farmer & $1(10)$ & $1(10)$ \\
\hline Hospital worker & 0 & $2(20)$ \\
\hline Merchant & $2(20)$ & $4(40)$ \\
\hline Student & $4(40)$ & 0 \\
\hline Housewife & $3(30)$ & $3(30)$ \\
\hline \multicolumn{3}{|l|}{ Education, n (\%) } \\
\hline Primary school & $6(60)$ & $4(40)$ \\
\hline Secondary school & 0 & $3(30)$ \\
\hline Bachelor's degree or higher & $4(40)$ & $3(30)$ \\
\hline Low back pain duration (month), mean (SD) & $46.7(47.7)$ & $41.6(27.8)$ \\
\hline \multicolumn{3}{|l|}{ Work status, n (\%) } \\
\hline Working full time & $10(100)$ & $9(90)$ \\
\hline Working part time & 0 & $1(10)$ \\
\hline \multicolumn{3}{|l|}{ Area of symptoms, n (\%) } \\
\hline Low back pain (LBP) & $7(70)$ & $6(60)$ \\
\hline LBP and leg pain above knee & $1(10)$ & $1(10)$ \\
\hline LBP and leg pain below knee & $2(20)$ & $3(30)$ \\
\hline \multicolumn{3}{|l|}{ Previous treatments, $\mathrm{n}(\%)$} \\
\hline Physical therapy & $1(10)$ & $1(10)$ \\
\hline Massage & $1(10)$ & $2(20)$ \\
\hline Medication & $8(80)$ & $7(70)$ \\
\hline
\end{tabular}

Table 2. Comparison of mean post-test measures at 10 weeks of intervention between the core stabilization exercise and control groups after adjustment for differences in baseline values using analysis of covariance (ANCOVA)

\begin{tabular}{lccc}
\hline \multirow{2}{*}{ Outcomes } & \multicolumn{3}{c}{ At 10 weeks of intervention } \\
\cline { 2 - 4 } & $\begin{array}{c}\text { Core stabilization exercise } \\
(\mathrm{n}=10)\end{array}$ & $\begin{array}{c}\text { Control } \\
(\mathrm{n}=10)\end{array}$ & $\begin{array}{c}\text { Difference } \\
(95 \% \mathrm{CI})\end{array}$ \\
\hline Pain intensity of instability catch sign & 1.81 & 4.89 & $3.09^{*}(1.53$ to 4.64$)$ \\
Roland Morris Disability Questionnaire & 3.36 & 7.43 & $4.07^{*}(1.66$ to 6.48$)$ \\
TrA \& IO/RA ratio & 10.40 & 2.51 & $-7.89^{* *}(-10.05$ to -5.74$)$ \\
LM/ICLT ratio & 0.75 & 0.38 & $-0.50(-1.07$ to 0.07$)$ \\
\hline
\end{tabular}

Note: $\operatorname{TrA} \& \mathrm{IO} / \mathrm{RA}$ ratio $=$ ratio activation of trasversus abdominis and internal oblique relative to rectus abdominis, LM/ICLT ratio $=$ ratio activation of lumbar multifidus relative to iliocostalis lumborum pars thoracis, *Significant difference between groups $(\mathrm{p}<0.01),{ }^{* *}$ Significant difference between groups $(\mathrm{p}<0.001)$

Sub-MVIC testing and ADIM showed high reliability with ICCs of $0.96-0.98$ and $0.96-0.98$, respectively.

When differences between the two groups were analyzed, significantly greater reductions in pain intensity during ICS ( $\mathrm{p}=0.001)$, functional disability scores $(\mathrm{p}=0.002)$ and higher TrA \& IO/RA ratios $(p<0.001)$ were found in the CSE group compared to $\mathrm{CG}$, while there was no significant difference between groups in the LM/ICLT ratio (Table 2).

\section{DISCUSSION}

The results of this study show the therapeutic effects of the 10 weeks of core stabilization exercise on pain intensity of the instability catch sign (ICS), functional disability, and trunk muscle activation patterns of patients with clinical lumbar instability. The findings of this study suggest that both CSE and hydrocollator therapy with trunk stretching exercises reduce pain intensity of ICS and decrease functional 
disability; however, improvement in the activation ratio of TrA \& IO relative to RA muscles (TrA \& IO/RA ratio) was found only in the CSE group.

CSE may enhance the ability of segmental muscles in the lower back, reducing the pain intensity of ICS and improved functional disability of patients with clinical lumbar instability. ICS is one of the major problems of clinical lumbar instability ${ }^{8,10,27)}$. It has been proposed that ICS is sudden sharp pain in mid-range of motion during return from a flexed trunk position ${ }^{6,27)}$. It is thought that the pain of ICS arises from degenerative osteoligamentous laxity, segmental stabilizing muscle dysfunction or reduced neuromuscular control $^{8,26)}$. Normally, movement of the lumbar spine during return from a flexed trunk position is smooth and painless; however, patients with clinical lumbar instability commonly show ICS, when they move from trunk flexion to an erect position. ICS may be caused by abnormal movement, such as increasing shear force on the affected lumbar motion segment, and this may irritate the pain receptors in the facet joints, vertebral ligaments or other soft tissues around the affected lumbar motion segment ${ }^{6)}$. Abnormal movement of the affected lumbar motion segment may eventually alter neuromuscular control leading to deficiencies in lumbar spinal stability ${ }^{26,28)}$. CSE provided significantly better reduction of pain intensity during ICS than stretching exercises and hydrocollator therapy, possibly because the deep abdominal muscles having achieved their preferential activations after 10 weeks of training, decreasing abnormal movement of the affected lumbar motion segment. It has been reported that an increase in activation of the deep abdominal (TrA \& IO) or deep back (LM) muscles may reduce pain intensity of the lower back and functional disability ${ }^{24)}$.

Patients in CSE showed a decrease in functional disability that may have been due to their reduced pain. This finding of the present study was in agreement with those of previous studies of patients with chronic non-specific $\mathrm{LBP}^{15}$ ), spondylolysis or spondylolisthesis ${ }^{12)}$. O'Sullivan et al. ${ }^{12)}$ stated that the abdominal drawing-in maneuver (ADIM) can provide powerful biofeedback to reinforce the integration of muscle control into functional tasks. Additionally, Cholewicki et al. ${ }^{29)}$ showed that ADIM serves to stabilize the lumbar spine during functional tasks healthy subjects. Stretching exercises and hydrocollator therapy also reduced pain intensity of ICS. Previous studies have shown that stretching exercise ${ }^{30)}$ and heat therapy ${ }^{31)}$ significantly decreased the pain intensity at 24 hours after treatments. However, many studies have suggested that the acute-effect of pain inhibition provided by stretching exercise and heat therapy does not last for a long period ${ }^{32,33)}$. Therefore, pain inhibition may have been the result of changes in tissue properties arising from stretching exercise and heat therapy. It has been suggested that stretching exercise and heat therapy influence spinal alignment during dynamic movement ${ }^{34}, 35$ ). Thus, the combined effects of stretching exercise and the hydrocollator over 10 weeks might have decreased reflexive muscle spasms, allowing optimal spinal movement, which resulted in reduction of ICS pain.

The CSE group showed an improvement in the TrA \& IO/ $\mathrm{RA}$ ratio. It may have been a specific training effect of CSE which focuses on preferential retraining of $\operatorname{TrA}$ and inferior fibers of IO muscles ${ }^{10)}$. Nevertheless, the present study failed to show an improvement in LM muscle activation after exercise intervention, although the LM muscle is known to provide lumbar segmental stiffness ${ }^{36)}$, and controls the lumbar segment during sustained posture and movements ${ }^{10}$, 37). This may be a result of using sEMG to record activation of the LM muscle. The recorded EMG signal probably represented activation of the superficial fibers of the LM muscle which produces torque to create trunk extension and spinal compression $^{38)}$. In contrast, the deep fibers of the LM muscle provide segmental stability in a nonspecific manner, similar to the TrA muscle ${ }^{38)}$. Therefore, the significant decrease in the $\operatorname{TrA} \&$ IO/RA ratio in the CG may have been due to insufficient stimulus of the TrA \& IO muscles during the stretching exercises. In fact, previous studies have shown the decrease in muscle force generating capacity after stretching exercise $^{39,40)}$ and heat therapy ${ }^{41)}$. Although the baseline values of the TrA \& IO/RA ratio were significantly different between the groups, this was addressed by ANCOVA to take account of this baseline difference in the statistical analysis. Further studies should use stratification to assure a valid comparison and balance among the outcome variables.

\section{ACKNOWLEDGEMENTS}

We thank all the participants in the study. We also thank Dr. Jeff Johns (Faculty of Pharmaceutical Sciences, Khon Kaen University) for proofreading. This study was supported by grants from the Back, Neck and Other Joint Pain Research Group and the Faculty of Associated Medical Sciences, Khon Kaen University, Thailand.

\section{REFERENCES}

1) Chou R, Huffman LH: Nonpharmacologic therapies for acute and chronic low back pain: a review of the evidence for an American Pain Society/ American College of Physicians clinical practice guideline. Ann Intern Med, 2007, 147: 492-504. [Medline]

2) Kaewduangdee $P$, Puntumetakul R, Boonprakob $Y$, et al.: The prevalence of musculoskeletal disorders in the textile occupation in Khon Kaen province. J Med Tech Phys Ther, 2010, 22: 292-301.

3) Kaewduangdee P, Puntumetakul R, Chatchawan U, et al.: Prevalence and associated risk factors of low-back pain in textile fishing net manufacturing. Hum Factors Ergon Manuf Serv Ind, (in press).

4) Thepdara S, Tiwaiyakornwilas $P$, Thani $P$, et al.: The improvement of drugs decreasing pattern in musculoskeletal system at NongKae Primary Care Unit Ubonratchathani Province. Thai J Phys Ther, 2005, 28: 1-10.

5) White AA, Panjabi MM: The problems of clinical instability in the human spine: a systematic approach. In: Clinical biomechanics of the spine. $2^{\text {nd }}$ ed. Philadelphia: JB Lippincott, 1990, p 278.

6) Kotilainen E, Valtonen S: Clinical instability of the lumbar spine after microdiscectomy. Acta Neurochir (Wien), 1993, 125: 120-126. [Medline] [CrossRef]

7) Hicks GE, Fritz JM, Delitto A, et al.: Inter-rater reliability of clinical examination measures for identification of lumbar segmental instability. Arch Phys Med Rehabil, 2003, 84: 1858-1864. [Medline] [CrossRef]

8) Paris SV: Physical signs of instability. Spine, 1985, 10: 277-279. [Medline] [CrossRef]

9) Iguchi T, Kanemura A, Kasahara K, et al.: Age distribution of three radiologic factors for lumbar instability: Probable aging process of the instability with disc degeneration. Spine, 2003, 28: 2628-2633. [Medline] [CrossRef]

10) Richardson CA, Jull GA: Muscle control-pain control. What exercises would you prescribe? Man Ther, 1995, 1: 2. [Medline] [CrossRef] 
11) Hides JA, Jull GA, Richardson CA: Long-term effects of specific stabilizing exercises for first-episode low back pain. Spine, 2001, 26: E243E248. [Medline] [CrossRef]

12) O'Sullivan PB, Phyty GD, Twomey LT, et al.: Evaluation of specific stabilizing exercise in the treatment of chronic low back pain with radiologic diagnosis of spondylolysis or spondylolisthesis. Spine, 1997, 22: 2959-2967. [Medline] [CrossRef]

13) Kumar SP: Efficacy of segmental stabilization exercise for lumbar segmental instability in patients with mechanical low back pain: a randomized placebo controlled crossover study. N Am J Med Sci, 2011, 3 456-461. [Medline] [CrossRef]

14) Macedo LG, Latimer J, Maher CG, et al.: Motor control or graded activity exercises for chronic low back pain? A randomized controlled trial. BMC Musculoskelet Disord, 2008, 9: 65. [Medline] [CrossRef]

15) Koumantakis GA, Watson PJ, Oldham JA: Trunk muscle stabilization training plus general exercise versus general exercise only: Randomized controlled trial of patients with recurrent low back pain. Phys Ther, 2005, 85: 209-225. [Medline]

16) Mannion AF, Balague F, Pellise F, et al.: Pain measurement in patients with low back pain. Nat Clin Pract Rheumatol, 2007, 3: 610-618. [Medline] [CrossRef]

17) Jirarattanaphochai $K$, Jung $S$, Sumananont $C$, et al.: Reliability of the Roland-Morris Disability Questionnaire (Thai version) for the evaluation of low back pain patients. J Med Assoc Thai, 2005, 88: 407-411.

18) Dankaerts W, O'Sullivan PB, Burnett AF, et al.: Reliability of EMG measurements for trunk muscles during maximal and sub-maxima voluntary isometric contractions in healthy controls and CLBP patients. Electromyogr Kinesiol, 2004, 14: 333-342. [Medline] [CrossRef]

19) Marshall P, Murphy BA: The validity and reliability of surface EMG to assess the neuromuscular response of the abdominal muscles to rapid limb movement. J Electromyogr Kinesiol, 2003, 13: 477-489. [Medline] [CrossRef]

20) Imai A, Kaneoka K, Okubo Y, et al.: Trunk muscle activity during lumbar stabilization exercises on both a stable and unstable surface. J Orthop Sports Phys Ther, 2010, 40: 369-375. [Medline]

21) Areeudomwong P, Puntumetakul R, Kaber DB, et al.: Effects of handicraft sitting postures on lower trunk muscle fatigue. Ergonomics, 2012, 55 693-703. [Medline] [CrossRef]

22) Danneels LA, Cagnie BJ, Cools AM, et al.: Intra-operator and interoperator reliability of surface electromyography in the clinical evaluation of back muscles. Man Ther, 2001, 6: 145-153. [Medline] [CrossRef]

23) Ng JK, Richardson CA, Parniapour M, et al.: EMG activity of trunk muscles and torque output during isometric axial rotation exertion: a comparison between back pain patients and matched controls. J Orthop Res, 2002, 20: 112-121. [Medline] [CrossRef]

24) O'Sullivan PB, Twomey L, Allison GT: Altered abdominal muscle recruitment in patients with chronic back pain following a specific exercise intervention. J Orthop Sports Phys Ther, 1998, 27: 114-124. [Medline]

25) Chanthapetch P, Kanlayanaphotporn R, Gaogasigam C, et al: Abdomina muscle activity during abdominal hollowing in four starting positions. Man Ther, 2009, 14: 642-646. [Medline] [CrossRef]
26) Silfies SP, Squillante D, Maurer $P$, et al.: Trunk muscle recruitment patterns in specific chronic low back pain populations. Clin Biomech (Bristol, Avon), 2005, 20: 465-473. [Medline] [CrossRef]

27) Fritz JM, Erhard RE, Hagen BF: Segmental instability of the lumbar spine. Phys Ther, 1998, 78: 889-896. [Medline]

28) Gardner-Morse MG, Stokes IA: The effects of abdominal muscle coactivation on lumbar spine stability. Spine, 1998, 23: 86-91. [Medline] [CrossRef]

29) Cholewicki J, Juluru K, Radebold A, et al.: Lumbar spine stability can be augmented with an abdominal belt and/or increased intra-abdominal pressure. Eur Spine J, 1999, 8: 388-395. [Medline] [CrossRef]

30) Ylinen J, Kautiainen H, Wirén K, et al.: Stretching exercise vs manual therapy in treatment of chronic neck pain: a randomized, controlled crossover trial. J Rehabil Med, 2007, 39: 126-132. [Medline] [CrossRef]

31) Steiner D, Ersala G, Hengehold D, et al.: Continuous low-level heat therapy for acute muscular low back pain. In: Proceedings of the 19th Annual Scientific Meeting of the American Pain Society, 2000, p 112.

32) Halbertsma JPK, Mulder I, GÖeken LNH, et al.: Repeated passive stretching: acute effect on the passive muscle moment and extensibility of short hamstring. Arch Phys Med Rehabil, 1999, 80: 407-414. [Medline] [CrossRef]

33) Reitman C, Esses SI: Conservative options in the management of spina disorders. Part I: bed rest, mechanical and energy-transfer therapies. Am J Orthop, 1995, 24: 109-116. [Medline]

34) Lewis SE, Holmes PS, Woby SR, et al.: Short-term effect of superficial heat treatment on paraspinal muscle activity, stature recovery, and psychological factors in patients with chronic low back pain. Arch Phys Med Rehabil, 2012, 93: 367-372. [Medline] [CrossRef]

35) Williams S, White CE, Hancock $T$, et al.: Comparison of repeated stretching and static stretching in extension of the lumbar spine in healthy adults. Int J Mech Diagn Ther, 2004, 8: 169-177.

36) Bogduk N, Macintosh JE, Pearcy MJ: A universal model of the lumbar back muscles in the upright position. Spine, 1992, 17: 897-913. [Medline] [CrossRef

37) MacDonald DA, Moseley GL, Hodges PW: The lumbar multifidus: Does the evidence support clinical beliefs? Man Ther, 2006, 11: 254-263. [Medline] [CrossRef]

38) Moseley GL, Hodges P, Gandevia S: Deep and superficial fibers of the lumbar multifidus muscle are differentially active during voluntary arm movement. Spine, 2002, 27: E29-E36. [Medline] [CrossRef]

39) Avela J, Kyr Öläinen H, Komi PV, et al.: Reduced reflex sensitivity persists several days after long-lasting stretch-shortening cycle exercise. J Appl Physiol, 1999, 86: 1292-1300. [Medline]

40) Evetovich TK, Nauman NJ, Conley DS, et al.: Effects of static stretching of the biceps brachii on torque, electromyography, and mechanomyography during concentric isokinetic muscle actions. J Strength Cond Res, 2003, 17: 484-488. [Medline]

41) Newton RA: Contemporary views on pain and the role played by thermal agents in managing pain symptoms. In: Thermal Agents in Rehabilitation. Philadelphia: FA Davis, 1990, pp 18-42. 